\title{
Efficacy of lymphatic sealing using the LigaSure in kidney transplantation
}

\author{
Sangkyun Mok, Sun Cheol Park, Young Jun Park, Sang Seob Yun, Jang Yong Kim
}

Department of Surgery, Seoul St. Mary's Hospital, College of Medicine, The Catholic University of Korea, Seoul, Korea

Background: lliac vessel lymphatic ligation is extremely important, as it is associated with the occurrence of lymphocele in kidney transplantation. Conventional silk tie ligation is mainly used in lymphatic ligation; however, the LigaSure (vessel-sealing device) is also effective for lymphatic ligation. This study aimed to evaluate the efficacy of lymphatic sealing using the LigaSure in kidney transplantation.

Methods: This retrospective study included patients who underwent kidney transplantation from the prospectively registered database at Seoul St. Mary's Hospital, South Korea, between January 1, 2020 and September 2, 2020. We analyzed comorbidities, primary renal disease, transplant variables, and post-transplantation outcomes.

Results: Seventy-one patients were enrolled in this study. The mean age of the patients was 48 years. The LigaSure and conventional groups comprised of $30(42 \%)$ and $41(58 \%)$ patients, respectively. There were no statistically significant differences in clinical characteristics except hypertension and induction agent. There was no difference in the occurrence of lymphoceles, postoperative day $1(P=0.810)$ and day $7(P=0.798)$ drain volume and drain removal length $(P=0.947)$ between the two groups during the observation period was observed.

Conclusions: The results of our study show that LigaSure was comparable with conventional lymphatic ligation. There was no difference in the postoperative outcomes. Therefore, LigaSure-based iliac lymphatic ligation can be safely used in kidney transplantation.

Corresponding author: Sangkyun Mok

E-mail: skmok81@gmail.com

(C) The Korean Society for Transplantation

This is an Open Access article distributed under the terms of the Creative Commons Attribution Non-Commercial License (http://creativecommons.org/licenses/by-nc/4.0/) which permits unrestricted non-commercial use, distribution, and reproduction in any medium, provided the original work is properly cited. 